\title{
A deep learning approach for prediction of SARS-CoV-2 cases using the weather factors in India
}

\author{
Kantha Rao Bhimala ${ }^{1}$, GOPAL KRISHNA PATRA ${ }^{1}$, Rajashekar Mopuri ${ }^{2}$, and Srinivasa \\ Rao Mutheneni² \\ ${ }^{1}$ CSIR Fourth Paradigm Institute \\ ${ }^{2}$ Indian Institute of Chemical Technology CSIR
}

October 15, 2020

\begin{abstract}
Advanced and accurate forecasting of COVID-19 cases play a crucial role in management of hospital facility, policy decision, logistic support, and economy of the country. Artificial Intelligence (AI) techniques have proved its capability in time series forecasting of the non-linear problems. The present study assessed the relationship between weather parameters and COVID-19 cases and found the specific humidity have strong positive association, maximum temperature have negative and minimum temperature have positive association in most of the states in India. Further, we have developed a weather integrated LSTM (long short term memory) models for advanced (1-14 days) forecasting of the COVID-19 cases over different states in India. To achieve the goal we have utilized the humidity and temperature time series data along with the COVID-19 confirmed cases data (1st April-30th June 2020) to optimise the LSTM model in univariate and multivariate modes. The optimised models are utilized to forecast the COVID-19 cases for the period 1st July, 2020 to 31st July 2020 with 1 to 14days lead time. The results shows that the univariate LSTM model (past COVID-19 input) have reasonably good skill (Relative Error $<20 \%)$ in short range forecast (1day lead) for most of the selected states, whereas the skill is degraded with the medium and long range forecast. The major finding of the current study is that the medium range (1-7days) forecasting skill is enhanced in some of the states with the weather integrated multivariate LSTM models. The states (Maharashtra, Gujarat, Rajasthan, Madhya Pradesh, Haryana, and Punjab) located in West and North West India region, humidity play a key role in enhancement of medium range forecasting skill of the LSTM model. It is also observed that the states located in high humid regions (Kerala, Tamil Nadu, and West Bengal) temperature plays a key role in model enhancement.
\end{abstract}

\section{Hosted file}

COVID-19 India_Climate-Manuscript-Final.pdf available at https://authorea.com/users/367428/ articles/486861-a-deep-learning-approach-for-prediction-of-sars-cov-2-cases-using-theweather-factors-in-india 


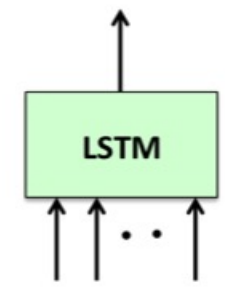

(a)

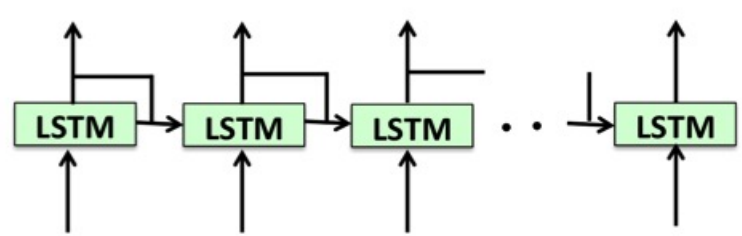

(b)

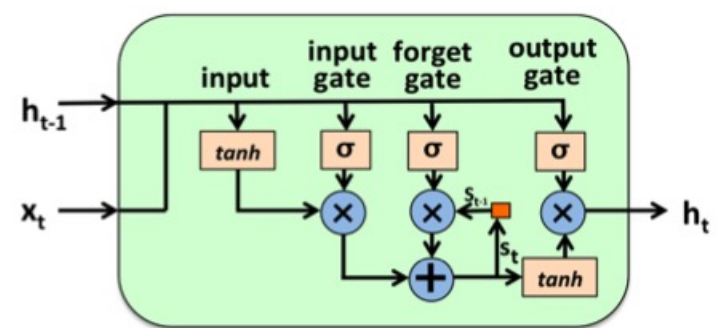

(c)
Structure of a gate

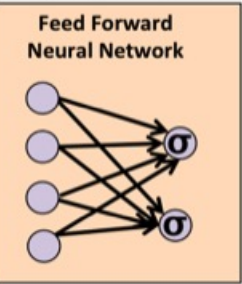

(d)

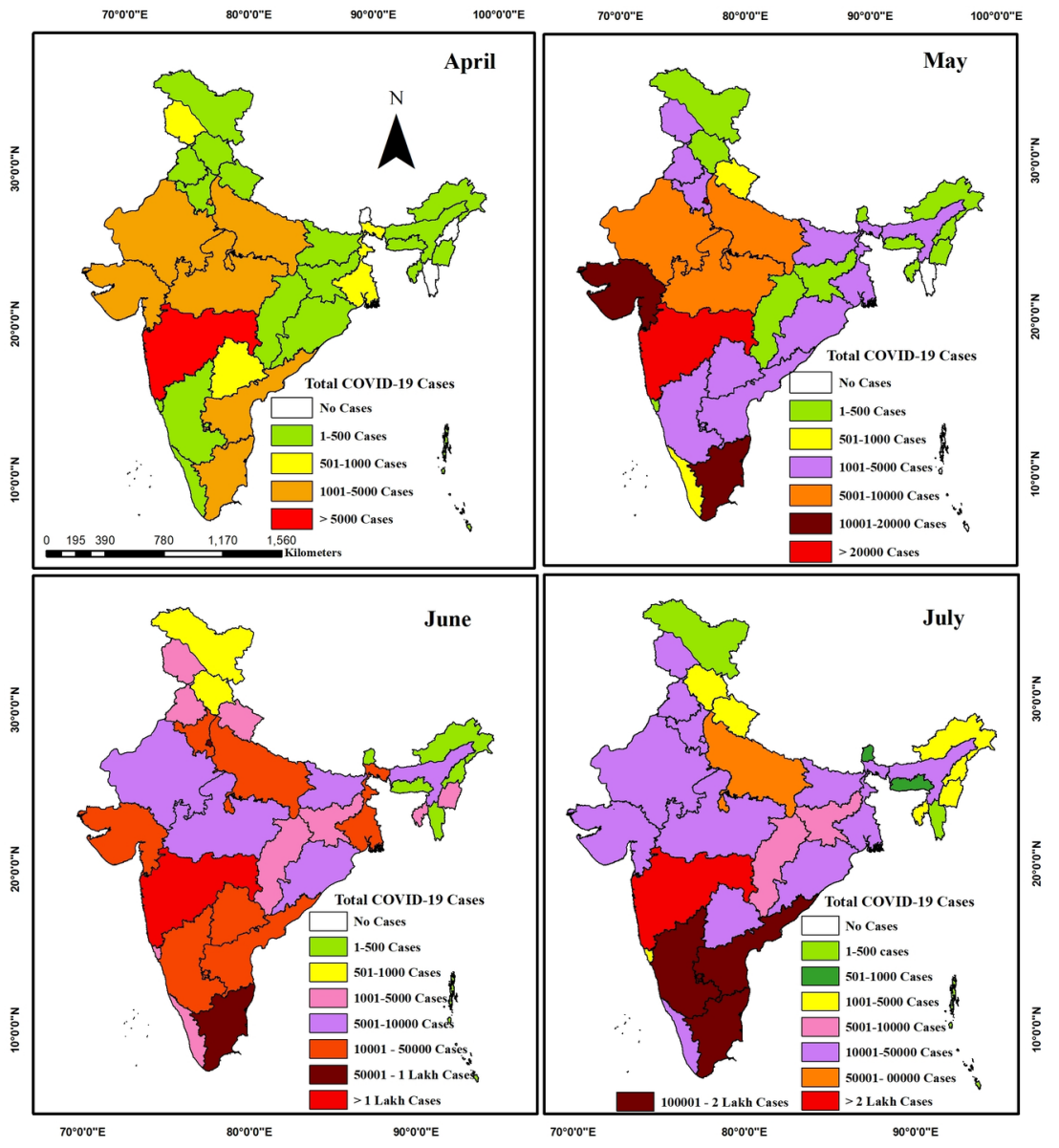



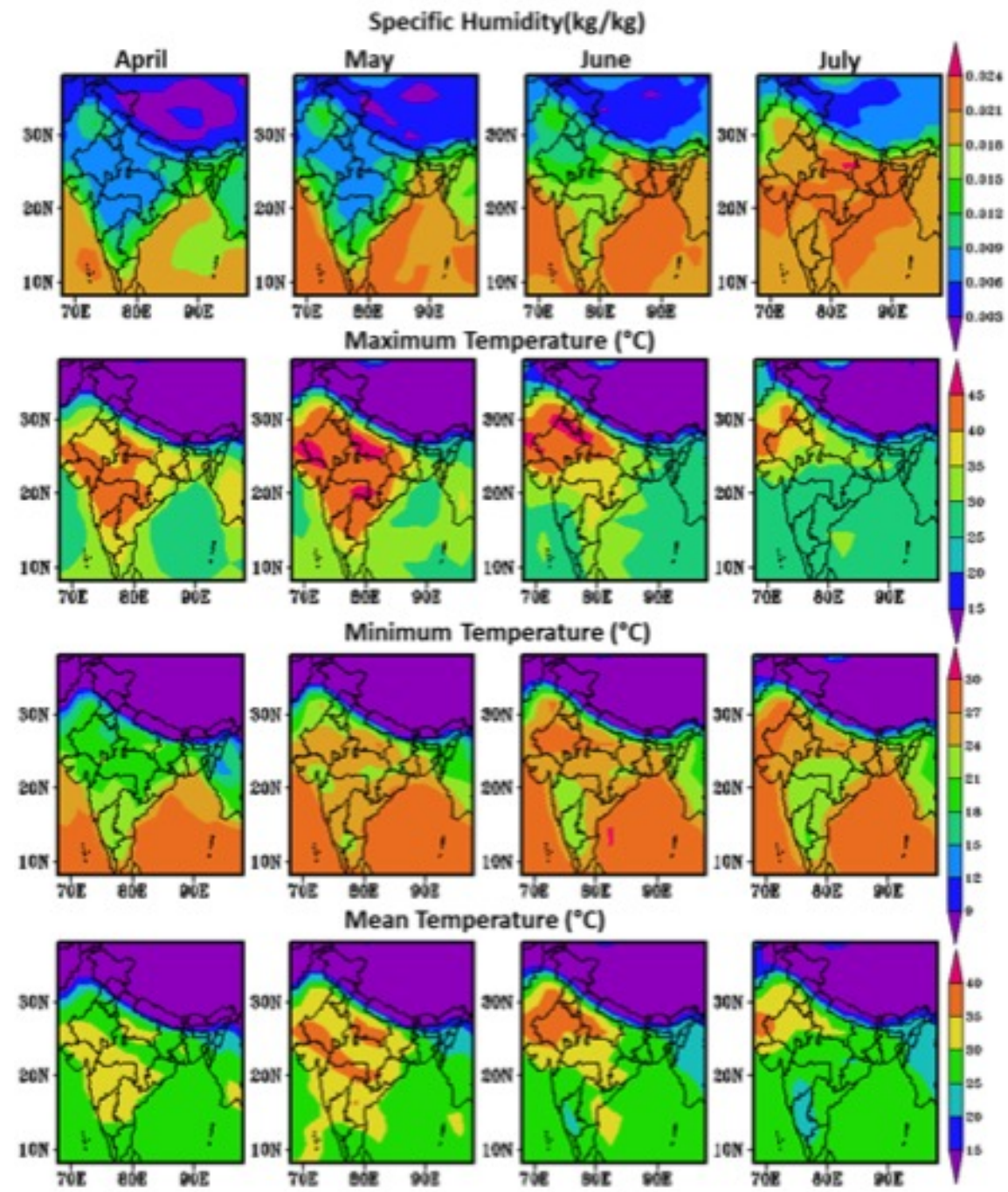
Correlation between COVID-19 cases and Specific humidity
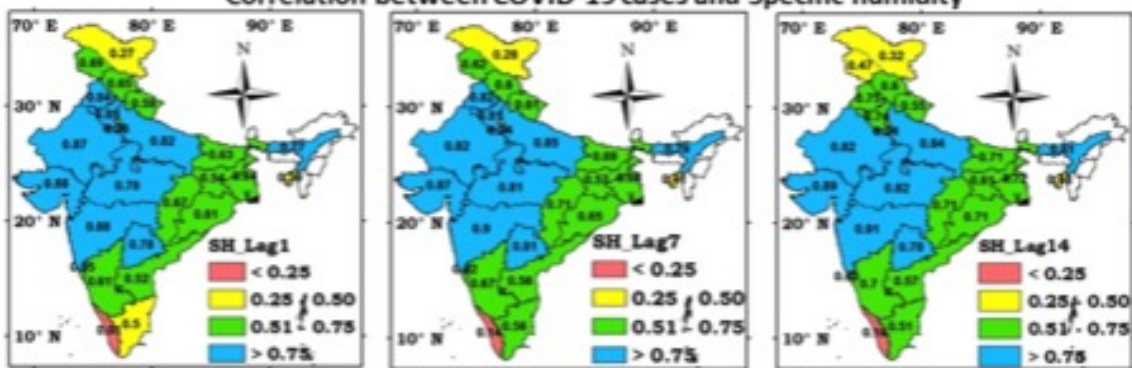

Correlation between COVID-19 cases and maximum temperature
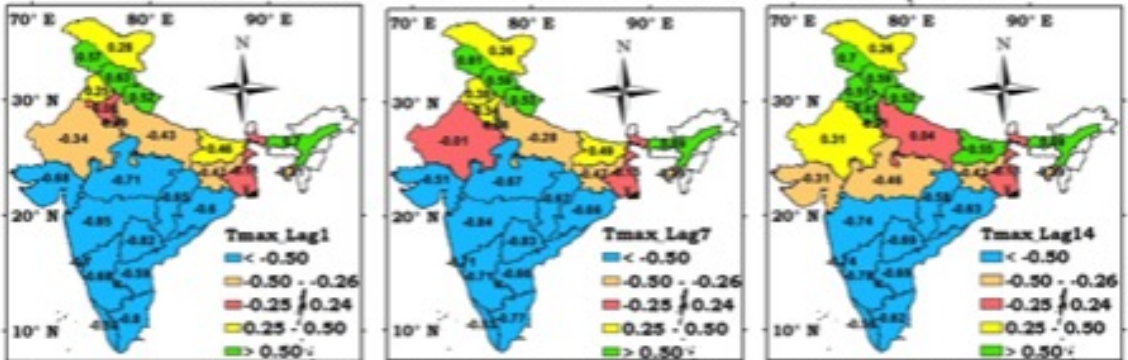

Correlation between COVID-19 cases and minimum temperature
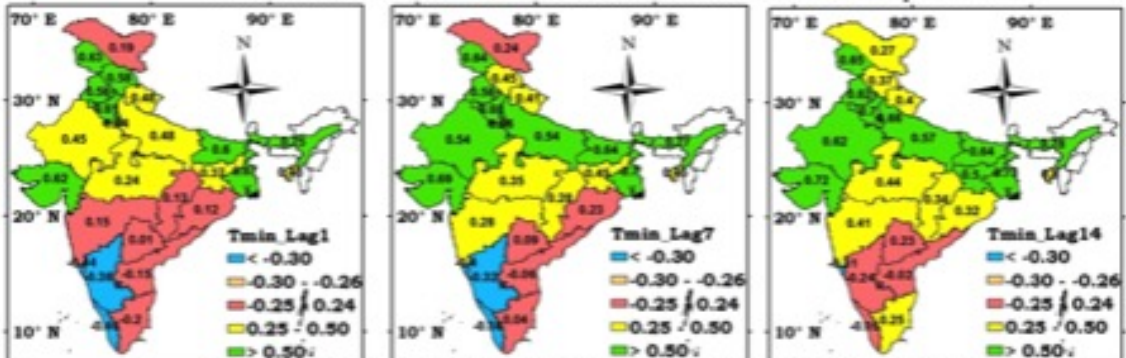

Correlation between COVID-19 cases and mean temperature
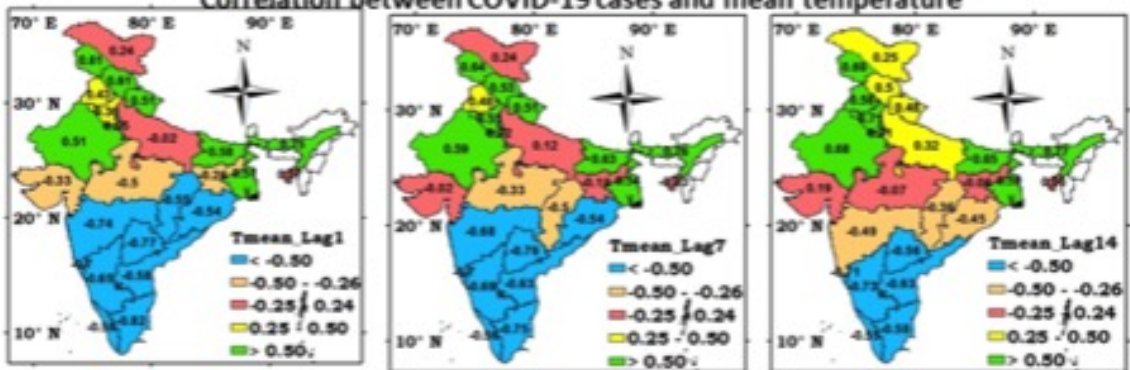


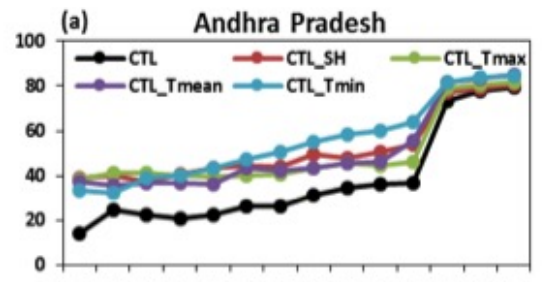

L1 L2 L3 LA L5 L6 L7 L8 L9 L10L11 L2 L13 L14

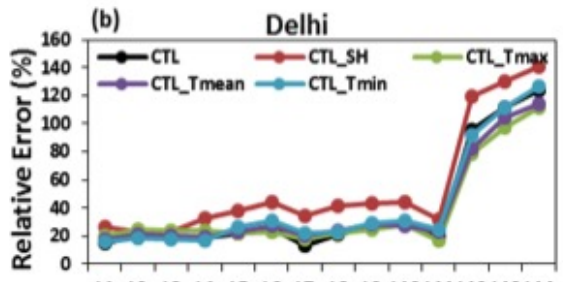

L1 L2 L3 L4 L5 L6 L7 L8 L9 L10L11L12 L13L14

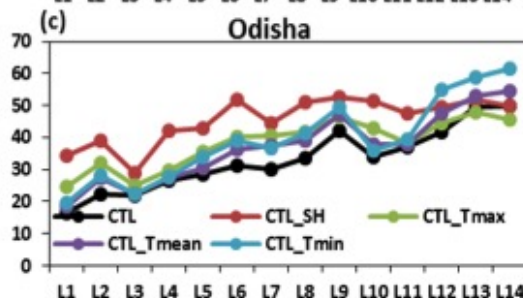

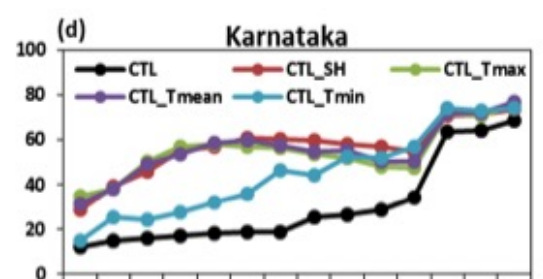

11 L2 L3 L4 L5 L6 L7 L8 L9 L10L11L12 L13 L14
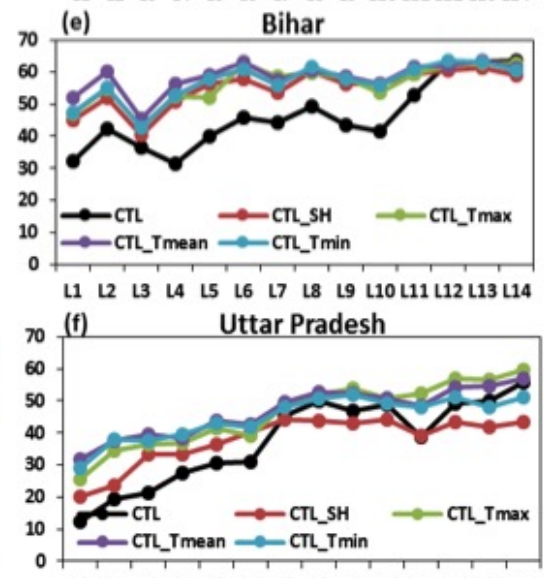

$\begin{array}{lllllllllllll}\text { L1 } & \text { L2 } & \text { L3 } & \text { L4 } & \text { L5 } & \text { L6 } & \text { L7 } & \text { L8 } & \text { L9 L10 L11 L12 L13 L14 }\end{array}$

Lag (days)
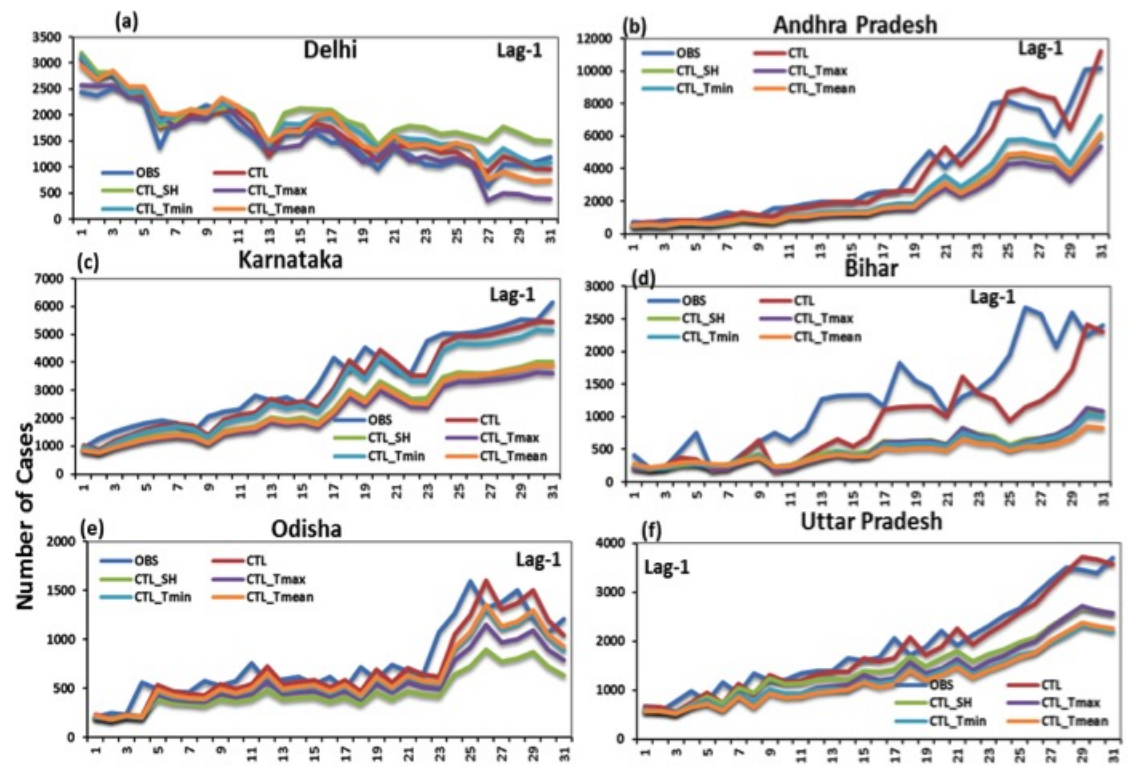

Days (starting from $01^{\text {st }}$ July 2020) 

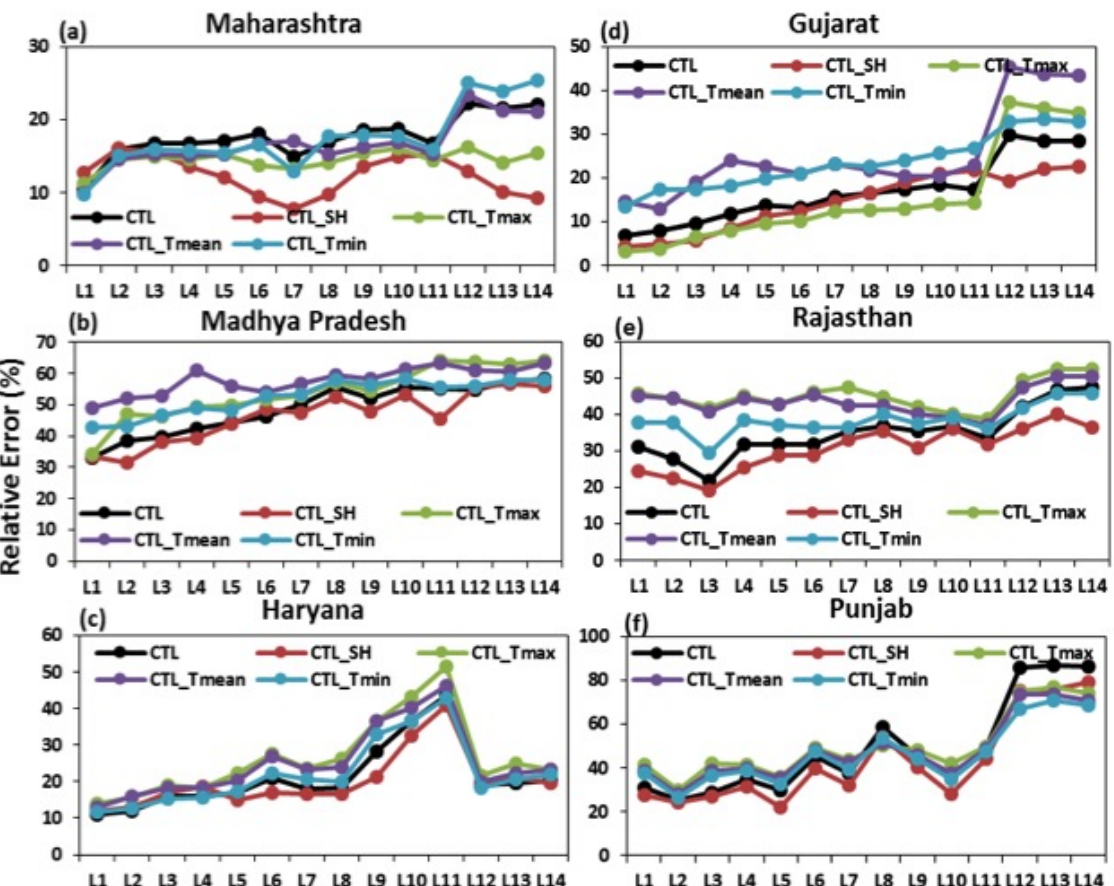

Lag (days)
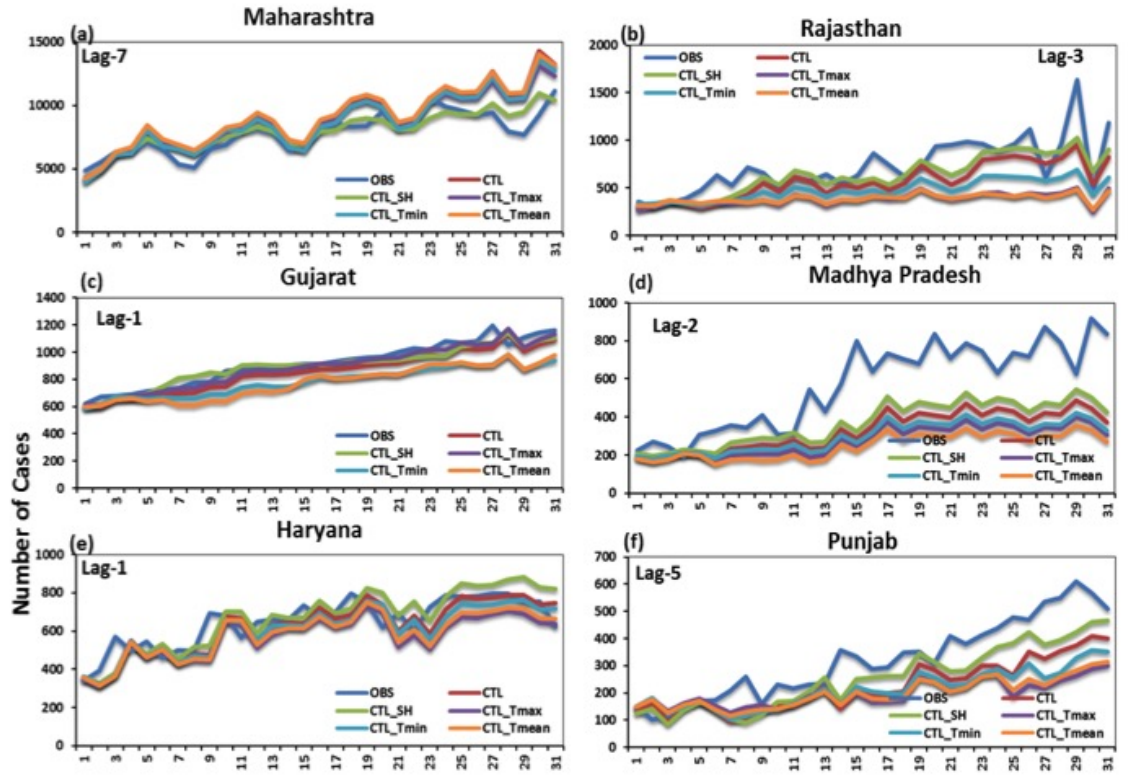

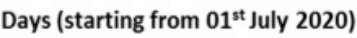



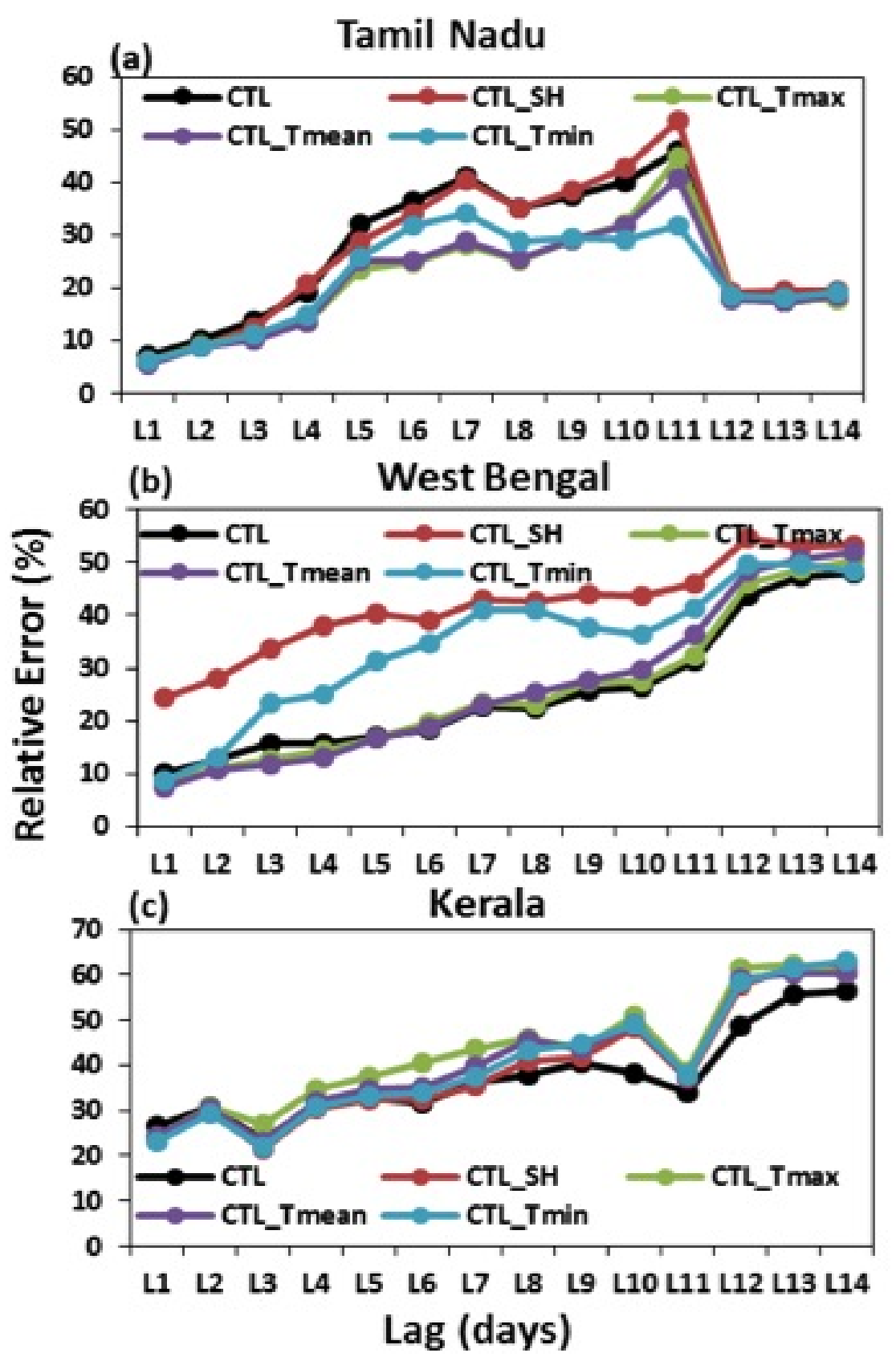

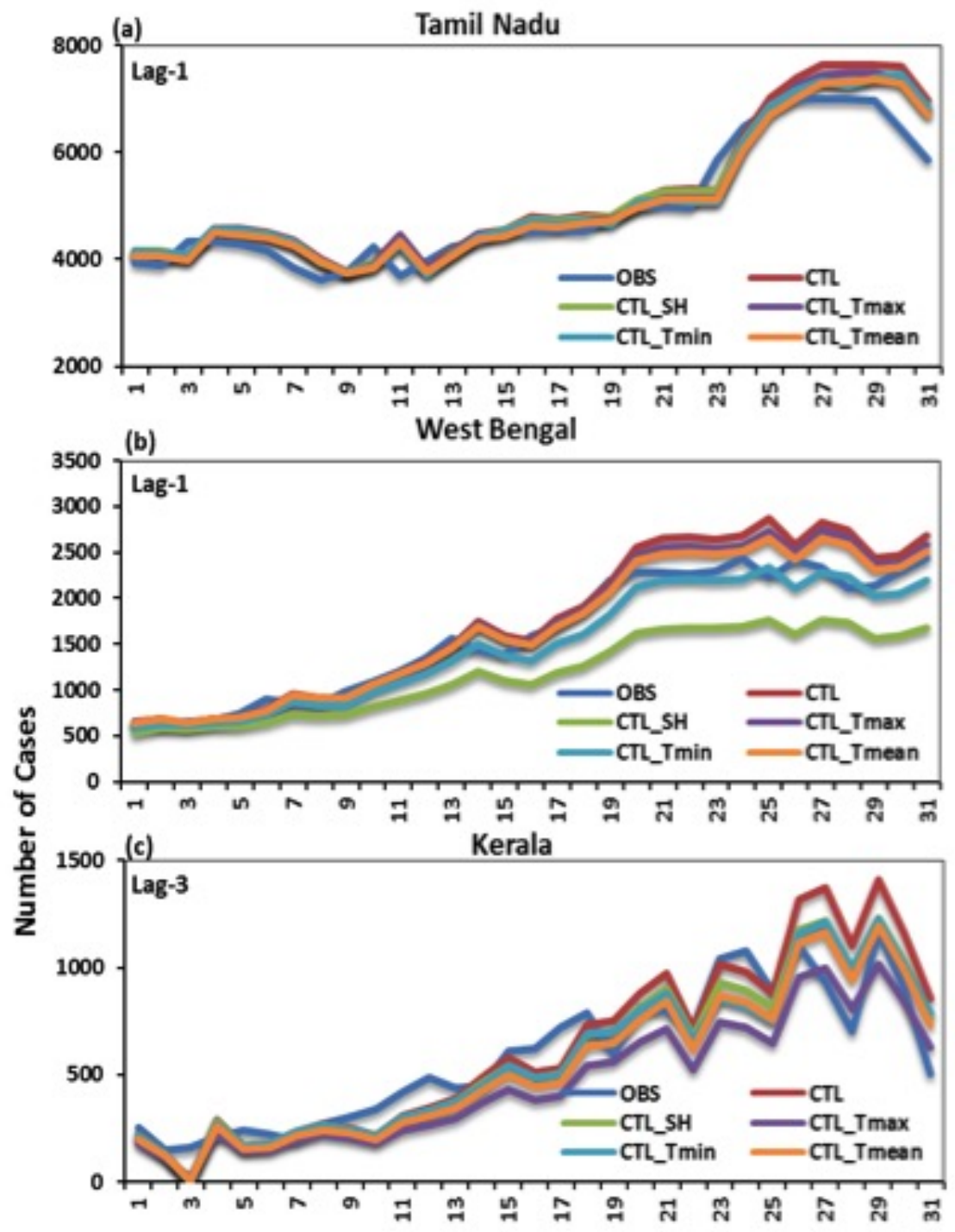

Days (starting from $01^{\text {st }}$ July 2020) 